\title{
THE ROLE OF 6-MINUTE WALK TEST (6MWT) AS A PROGNOSTIC MARKER IN SYSTEMIC SCLEROSIS
}

Vinicius Verlangieri Soubihe ${ }^{1}$, Juliana Zonzini Gaino ${ }^{1}$, Luciana Campanatti Palhares ${ }^{1}$, Marília Paula Souza dos Santos ${ }^{1}$, Zoraida Sachetto ${ }^{1}$, Ana Paula Toledo Del Rio1,*

1. Universidade Estadual de Campinas, Campinas (SP), Brazil.

*Corresponding author: anapauladelrio@hotmail.com

\section{BACKGORUND}

Respiratory complications of the systemic sclerosis (SSC) as pulmonary hypertension and interstitial lung disease (ILD) have become the leading causes of mortality among SSc patients. The detection of these complications and the assessment of their severity is of great interest and many tools are used as the 6-minute walk test (6MWT). It is a straightforward test frequently used in daily practice; however, its relevance in SSc is not well established. In this study, we aimed to evaluate the association between which one of 6MWT parameters and disease aspects known to be related with clinical worse prognosis.

\section{MATERIALS AND METHODS}

In this cross-sectional study, 38 SSc patients from the outpatient clinic of the rheumatology division of a tertiary unit were assessed regarding clinical data [disease duration, calcinosis, diffuse or limited, the New York Heart Association (NYHA) functional classification, autoantibodies (antitopoisomerase I, anticentromere, anti-RNP], interstitial lung disease (ILD) in thoracic computed tomography and C-reactive protein (CRP). Subjects were submitted to spirometry and to the $6 \mathrm{MWT}$. Six-minute walk test parameters (total distance, predicted distance, final-initial peripheral oxygen saturation and final Borg score) were compared with clinical data, immunological profile, presence of ILD, CRP, forced vital capacity through Kruskal-Wallis test and Spearman correlation coefficient with a $5 \%$ level of significance.

\section{RESULTS}

Among 6MWT items, total distance was shorter in females ( $p=0.0097)$ and in NYHA functional class II, III and IV ( $p=0.001)$. It was also negatively correlated with age $(r s=-0.449, p=0.004)$ and age at diagnosis ( $r s=-0.450, p=0.004)$. Predicted distance $(\%)$ was associated with forced vital capacity $(<I ; \mid<I I I$ and IV) $(p=0.006)$. Six-minute walk test items correlated differently with clinical, immunological, pulmonary and functional parameters. Total distance seems to be negatively correlated with age and age at diagnosis. Predicted distance is better related with forced vital capacity and peripheral oxygen saturation with diffuse cutaneous disease and elevated CRP, suggesting extensive lung disease. Final Borg score was associated with antitopoisomerase I positivity, a marker of a worse disease.

\section{CONCLUSION}

Our findings suggest that 6MWT is a performance test easy to apply in clinical practice with an important role at identifying patients with adverse outcomes, specially using walking distance, peripheral oxygen saturation and total Borg as the main parameters of this test. 